\title{
Using ABM to clarify and refine social practice theory
}

\author{
Kavin Narasimhan, Thomas Roberts, Maria Xenitidou and Nigel Gilbert \\ Centre for Research in Social Simulation (CRESS), Department of Sociology, \\ University of Surrey, Guildford GU2 7XH, UK \\ $\{\mathrm{k}$.narasimhan, t.m.roberts,m.xenitidou,n.gilbert\}@surrey.ac.uk
}

\begin{abstract}
We use an agent-based model to help to refine and clarify social practice theory, wherein the focus is neither on individuals nor on any form of societal totality, but on the repeated performances of practices ordered across space and time. The recursive relationship between social practices and practitioners (individuals performing practices) is strongly emphasised in social practice theory. We intend to have this recursive relationship unfold dynamically in a model where practitioners and social practices are both considered as agents. Model conceptualisation is based on the principle of structuration theory - the focus is neither on micro causing macro nor on macro influencing micro, but on the duality between structure (macro) and agency (micro). In our case, we conceptualise the duality between practitioners and practices based on theoretical insights from social practices literature; where information is unclear or insufficient, we make systematic assumptions and account for these.
\end{abstract}

Keywords: social practice theory, modelling social practices, agentbased model, structuration theory

\section{Introduction}

In this paper we seek to clarify the core principles of social practice theory using an agent-based model. Existing information regarding the dynamics and growth of social practices ${ }^{1}$ are very rich but quite dense in that they are open for multiple interpretations. For instance, Kuijer provides an interesting tabular summary of the evolution of bathing as a social practice between 500 BC (the Roman empire) and the 1970s [1, p. 110]. But an exact pattern for the growth trajectory of the bathing practice is not available in current literature. It is a similar case for many other social practices - there is rich evidence suggesting that practices evolve over time but there is no analytical understanding of how this happens. We propose an agent-based model conceptualisation, a recognized method to formalise theoretical insights [2], to understand the mechanisms underlying the evolution of social practices. It is our aim that a model implemented based on

\footnotetext{
1 The terms 'practices' and 'social practices' are used interchangeably.
} 
our conceptualisation would be able to account for the evolution of practices in a systematic fashion. The rest of this paper is structured as follows. We provide an overview of social practice theory in section 2, which leads to identifying three specific processes we have chosen to conceptualise in section 3 . The actual model conceptualisation is then introduced in section 4 , followed by conclusions in section 5 .

\section{Overview of Social Practice Theory}

Social practice theory draws on many of the core principles of Gidden's theory of structuration [3], which considers that human activity and the social structures which shape it are recursively related. Shove notes that human activities are shaped and enabled by social structures of rules, and in turn, the structures are reproduced through human action [4]. In social practice theory, the focus is neither on individuals nor on any form of societal totality but on individuals performing practices that are ordered across space and time. Individuals are still a part of the social system, but the starting point for understanding social systems is the performance of practices by individuals (also referred to as practitioners).

Performing a social practice refers to the routine accomplishment of what people consider to be the normal ways of life [5, p.117]. Individuals are seen as carriers of practice; they carry out various tasks and activities that practices require [6]. This does not mean that individuals are regarded as passive beings [3], at the same time they are also not active in the sense of being involved in conscious decision-making [7]. Instead, individuals are considered to be skilled agents who actively negotiate and perform practices in the course of their daily lives. The 'social' status of a practice is then more a consequence of its stable reproduction beyond the limits of space, time and single individuals.

Practices are performed when all the relevant component elements are linked together [8, in review]. There is some debate about the nature of these different

elements, but there is a growing consensus around Shove's understanding of practices being made up of three core element groups: materials, meanings and skills [5]. As a minimum, one element from each of the three categories is required for a performance of the practice to occur. Through repeated performances, practices are reproduced across space and time. Practices can also evolve and/or eventually die out as the component elements change.

Practices rarely occur in isolation; they come together as bundles to make up lifestyles or habitus. For example, doing the laundry combines a number of individual practices such as loading and unloading the washing machine, drying the clothes, ironing and storing laundered clothes etc. (see figure 1). While these all remain separate practices, they are often performed together, leading to them sharing a common label.

Bundles (or practices) are also linked to each other. As the practices and elements in one bundle change there is a knock on effect for other related bundles. This process is known as co-evolution [8, in review]. For example, as the number of entertainment and wireless communication devices, such as televisions, laptop 


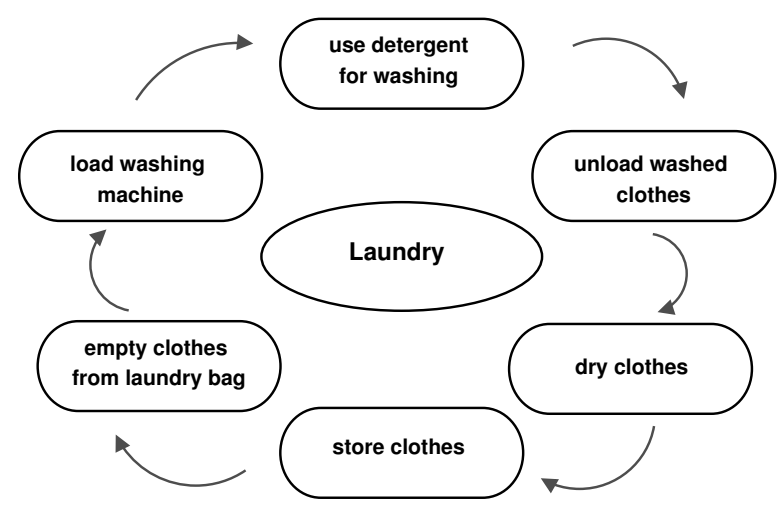

Fig. 1. Practices contributing to the laundry bundle

computers, tablets and smart phones, increases the practices associated with the use of these devices become less spatially constrained. This has led to the dispersal of such activities around the home with implications for other bundles of practices such as heating. Whereas in the past the whole family might spend an evening watching television together in the living room only needing to heat one room, today family members could be dispersed around the house independently watching, playing or communicating on multiple devices necessitating the need to heat the whole house. Similarly, as daily lives become more dependent on $\mathrm{ICT}^{2}$, an increasing number of bundles of practices become connected through the skills required to operate ICT equipment - e.g., using a computer for food preparation (using the internet to find a recipe) and communication (sending an e-mail). Shared meanings also link practices together, for example the desire for privacy while undertaking activities such as showering and using the lavatory.

\section{$3 \quad$ Identifying key model processes}

Gilbert defines target as the social phenomenon or process that an agent-based model seeks to represent [9]. In our case, demonstrating the evolution of social practices is the target phenomenon. From the social practices literature, we have identified three specific processes that contribute to this target phenomenon. First of these is the coming together of elements to enable the performances of practices. Shove suggests that meaning, material and skill elements come together to signify the performance of practices [10]. Elements initially exist in isolation waiting for links to be made, when this happens, practices come to exist. On the other hand, when elements are no longer linked, practices become obsolete and eventually cease to exist. Just as elements come together to form practices, loosely associated practices come together to form bundles, e.g., the aforementioned laundry example. In our model conceptualisation, we consider

\footnotetext{
${ }^{2}$ Information and Communications Technology
} 
both the coming together of elements to form practices as well as the coming together of practices to form bundles.

The second aspect we consider as contributing to the growth of social practices is the recursive relationship between practitioners and practices. The relationship signifies how practitioners perform practices, and how with repeated performances practices become established and widespread, i.e., become norms that feedback into future performances. Shove suggests an example to illustrate this concept [10]. Showering as a social practice has rapidly evolved during the last fifty years, and in this time, it has become a societal norm for people to shower everyday. This norm has influenced the practice of showering in different ways - new materials (e.g., soaps, bath gels), new skills (e.g., ability to use shower units) and new meanings (e.g., personal hygiene standards) have emerged. Figure 2 demonstrates the coming together of elements constituting the modern day practice of showering. The recursive relationship between practitioners and practices signifies how, in one direction micro-level phenomena (coming together of elements) leads to macro-level phenomena (the performance of practices). In the other direction, it signifies how practices afford the norms for their performances.

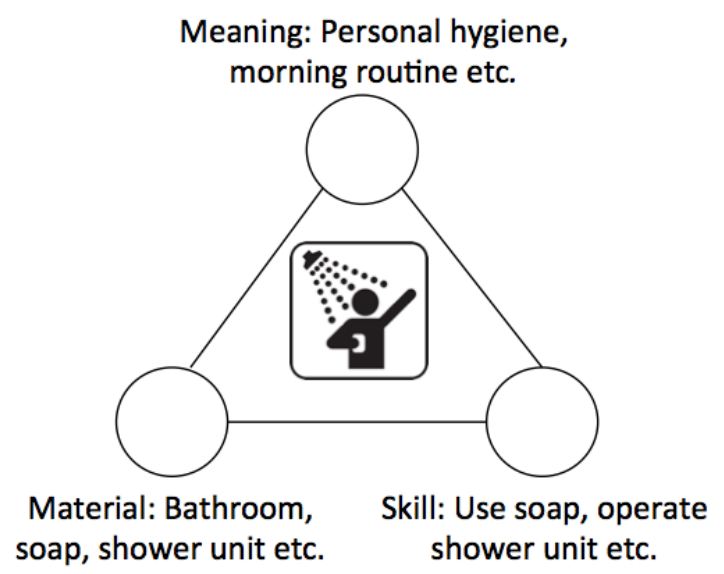

Fig. 2. Elements underlying the social practice of showering

Lastly, we consider the linked performances of practices, aka the co-evolution of practices. Co-evolution is the result of two or more practices evolving at the same time as a consequence of elements being shared between them. For instance, as noted in section 2, the growth of visual entertainment and ICT devices have influenced the evolution of heating practices in households.

Existing literature does not provide adequate details for modelling the processes we have identified here. For instance, there is theoretical and illustrative clarity of the recursive relationship between practitioners and practices, but there is no analytical understanding of the actual mechanisms involved. There 
is no explanation of rules and conditions under which elements come together (i.e. become linked) or disaggregate to influence the performances of practices. Likewise, there is no evidence suggestive of practices influencing norms for their performance. There is also a lack of understanding of mechanisms causing the co-evolution of practices (and bundles ${ }^{3}$ ). By systematically accounting for the missing details, we intend for our model conceptualisation to:

1. Demonstrate the coming together of elements to enable the performance of practices; demonstrate the coming together of practices to signify the performance of bundles.

2. Delineate the recursive relationship between practices and practitioners in an analytical fashion.

3. Demonstrate the co-evolution of practices.

\section{Conceptualisation of the model}

\subsection{Basic principles}

We consider four main entities in our model: elements, practitioners, practices and bundles. The last three entities are agents in the model, while elements (i.e., meaning, material and skill) are objects used by practitioners ( $h \_m e a n i n g$, $h \_m a t e r i a l$ and $\left.h \_s k i l l\right)$ and practices (p_meaning, p_material and $\left.p \_s k i l l\right)$, respectively. Practitioners are agents that perform practices, e.g., in the context of the laundry and showering examples considered above, households can be regarded as practitioner agents ${ }^{4}$. We consider practices as agents in the model to demonstrate the co-evolution relationship, which entails interaction between practices causing them to share elements. Consequently, we treat practices as agents that are capable of interacting with one another, to share and adapt their elements as an outcome of those interactions. Lastly, we consider bundles as agents that emerge in the model when two or more relevant practices come together.

Through the following example we demonstrate the relationship between the agents (households as practitioners, drying clothes as the social practice and laundry as the bundle). Household agents draw at least one element each (i.e. one $h \_m e a n i n g$, one $h \_m a t e r i a l$ and one $\left.h \_s k i l l\right)$ to perform the social practice of drying clothes. The exact elements chosen to perform the practice can differ across households. E.g., one can use tumble dryer as the material, while another uses cloth airer and some other household uses a radiator drying rack. This is an example of three different $h$ _material elements used by three different household agents. Despite differences in the actual elements used, all households

\footnotetext{
${ }^{3}$ Co-evolution applies to both practices and bundles.

4 Technically, it is individuals living within households who perform the practices. Since we like to consider several practices performed within the bounds of the household, even if different people perform them, we conceptualise households as the practitioners instead of individuals.
} 
intend to perform the same practice, i.e., dry clothes. Hence it is possible to say that the social practice of drying clothes has three different p_material elements contributing to its performance. On any particular occasion, if a household agent performs two or more associated practices, e.g. wash clothes, dry clothes and iron clothes, it is then considered to perform the laundry bundle. The specific roles of practitioners, practices and bundle agents are further detailed in section 4.3 after reviewing the general characteristics of agents and their interactions in section 4.2 .

\subsection{General characteristics of agents and their interactions}

Before we present the distinct properties of the practitioner, practice and bundle agents in section 4.3, we here present their common characteristics. Per Macy and Willer's recommendations, the agents are simple, autonomous, interdependent and adaptive [11]:

- Agents are simple in that they follow simple rules. Practitioners follow simple rules by way of performing practices out of habit. The assumption is based on the understanding of social practice theory that actors perform practices not as a consequence of conscious decision-making processes but mostly out of habit. Likewise, practice agents also follow simple rules to influence the norms for performances. As there are no theoretical insights of how bundles influence norms for future performances, we hypothesise that bundles influence norms by aggregating (a simple additive aggregation) the individual norms imposed by contributing social practices. Consider the laundry example in section 2. To determine norms imposed by laundry as a bundle, we propose computing the aggregate of norms imposed by each individual social practice contributing to the laundry bundle (loading the washing machine, unloading the washing machine, iron clothes etc.).

- Agents are interdependent in the sense that their actions affect one another. Interdependency of agents in the model can be explained at two levels. At one level, the coming together of material, meaning and skill elements facilitates the performance of practices, which in turn influences the norms affecting future performances of practices. At another level, the coming together of practices facilitates the production and reproduction of bundles; these in turn lead to norms (at the bundle level) affecting future performances of practices contributing to bundles.

- Agents are autonomous in the sense there is no global authority (entity) directing practitioners to perform practices; instead, they emerge purely from interactions at the micro-level. Elements referenced by practitioners come together to enable them to perform practices. Two or more practices come together to enable performances of bundles. In the other direction, practices and bundles influence norms for future performances of practices. These norms act as, in what [11] refers to as additional environmental constraints influencing the actions pursued by practitioners with regards to performing practices. However, constraints do not directly cause a practitioner to perform any practice. 
- Agents are adaptive: Practitioners modify performances based on the influences of existing practices and bundles. Each individual practitioner utilizes locally available information to perceive about and react to the norms imposed by the practice and bundle agents in its environment. Likewise, each individual practice (and bundle) agent uses locally available information to update its elements based on current performances by practitioners and the co-evolution links between practices.

There are three levels of interaction between model entities: micro-, mesoand macro-level interactions. Interactions between material, meaning and skill elements referenced by practitioners signify the micro-level interactions. These interactions cause elements to be updated, new links to be formed between elements, or existing links to be broken. Recollect that performances of practices signify the coming together of at least one meaning, one material and one skill elements. So, when micro-level interactions cause the meaning, material and skill elements essential to perform a practice to be linked, it enables a practitioner to perform that practice. In addition to that, when micro-level interactions between elements enable a practitioner to perform two or more social practices contributing to the same bundle, it leads to the performance of that bundle.

At a next level, interactions that occur among practitioner agents are classified as meso-level interactions. For instance, a practitioner agent assesses the performance of a particular practice by other practitioners in its social circle, and uses that as a social influence to modify or adapt its own performance of that practice. Meso-level interactions influence practitioners to modify the elements they reference and to form new links or disintegrate existing links between elements. Lastly, macro-level interactions signify the interactions between practices as a result of co-evolution. These interactions cause practices to modify the elements they reference or establish new links or disintegrate existing links between elements referenced across practices.

\subsection{Specific characteristics of model entities}

We now provide a detailed description of the distinct characteristics we have conceptualised for each model entity by considering as an example the performance of social practices within households:

- Elements represent three distinct categories of model entities: meaning, material and skill. Elements are objects used by practitioners and practices. Practitioners have access to any number of material, meaning and skill elements. Similarly, practices can be made up of any number of material, meaning and skill elements. But for the sake of model simplicity, we have assumed that in order to perform one practice, practitioners draw one element each, i.e., one material, one meaning and one skill elements.

- Practitioners are agents that are able to carry out practices. To do this, they have access to elements required to perform practices, but there is no evidence for what causes them to draw elements together. So we hypothesise 
that a variety of influences act upon practitioners and enable them to combine elements and carry out practices. The influences to be considered will vary depending upon the characteristics of the practitioners and practices being modelled. For instance, when households are the practitioners, we consider social influences and historical influences to impact the performance of practices. Social influences enable a household to carry out practices that are commonly performed in its social neighbourhood. Based on Reckwitz' (2002) observation, we consider historical influences to enable households to repeat their previous performances of practices [6]. While the influences act upon households causing them to update their elements (i.e. h_material, $h \_m e a n i n g$ and $\left.h \_s k i l l\right)$, they do not directly force households to perform specific practices.

- Practices are agents that exist from the start of the simulations, but may not be instantiated as performances. However, when practitioners start drawing elements together, practices are instantiated as performances and continue to exist as such, so long as practitioners keep repeating performances. If practices are not being performed, they become obsolete and may eventually cease to exist. Each practice agent maintains a list of meaning, material and skill elements contributing to its performance. During the simulation, these elements are updated based on two types of influences; the first one causes practices to update their list of elements based on current trends in how practices are performed and the elements used. E.g., if households adopt a new material or skill element for washing clothes, then the wash clothes social practice and laundry bundle update their respective lists of elements to include the newly adopted element. The second influence referred to as update based on co-evolution, causes practices to update their elements as a consequence of their relationship with one another. For instance, browsing the Internet is an ICT social practice, but at the same time, it can also serve as a skill element associated with the cooking practice for finding recipes. Given this relationship, if one practice (browsing the Internet) adopts a new material (using tablet PCs), then the other practice (cooking) also adopts that new element (tablet PCs to find recipes).

- Bundles: Shove suggests that practices come together to constitute bundles [10], but there is no evidence for what causes the coming together of practices. So we hypothesise establishing pre-defined soft links ${ }^{5}$, and then, to check if practitioners are in a position to perform these linked practices. If they are, then a new bundle agent emerges in the model. E.g., let us consider thermal control as a bundle that includes soft links between two social practices - use central heating and do thermal retrofits. Despite being soft linked, a household will be able to perform both practices only if influences acting upon the household should allow it. For instance, if pri-

\footnotetext{
${ }^{5}$ We define soft links as associations between practices (i.e., each practice linked to one or more other practices) defined at the start of simulations.
} 
vately rented, then households cannot perform thermal retrofits ${ }^{6}$, whereas households whose tenure is owned can. So in the former case, households can only perform a practice (i.e., use central heating), whereas in the latter case households can perform a bundle (i.e., thermal control).

- Environment: We conceptualise the model environment as a virtual society where practitioners, practices and bundles exist. The model environment has variables such as temperature, day of the week and season. A key principle of social practice theory is that the performances of practices are ordered across space and time [4]. So we hypothesise defining space-time boundaries as environmental influences acting upon practices and causing them to adapt their elements. For instance, when the scope of modelling extends between households and work places, then certain practices can be limited to performance within households (cooking, showering, etc.), while certain practices are restricted to be performed only outside the physical boundary of households (e.g., driving). Similarly, the performances of practices can also be restricted based on time - time of the day (morning, afternoon, evening and night) and season. E.g., it is unlikely home heating will be required during summer months, so the seasonal influence affects the meaning, material and skill elements needed to perform the central heating practice. This in turn imposes different norms during different seasons for households to perform the central heating practice.

\subsection{Conceptualising key processes}

Conceptualising the coming together of elements to enable the performances of practices: We hypothesise a similarity between elements rule that serves as a checkpoint to enable practitioners to perform practices. Since there is no evidence suggesting how elements come together to enable performing practices, we propose the following strategy as a sample approach. Compute an index of similarity between elements referenced by a practitioner and a practice, which if exceeds a desired threshold enables performing the desired practice. The strategy could be formulated in the following manner. Consider each element being represented as a bit string, e.g., [0110110111]. Compute pair-wise similarity between corresponding elements referenced by a practitioner and a practice. This will be the difference between 10 (i.e., bit string length) and the Hamming distance between elements. If the average pair-wise similarity (i.e., average of similarity measures between meaning, material and skill elements) exceeds a desired threshold, then a practitioner performs the desired practice.

Conceptualising the coming together of practices to enable the performance of bundles: Given the similarity between elements rule, we hypothe-

\footnotetext{
${ }^{6}$ In reality, housing tenure impacts an household's ability to undertake a thermal retrofit - a process to improve the thermal properties of a building through the use of high levels of thermal insulation and airtightness. Such a process will be much easier for people who own their homes than for those who live in privately rented properties. But for the sake of model simplicity, we have assumed that households residing in privately rented properties will not be able to do thermal retrofits.
} 
sise that practitioners are able to perform a bundle if they are able to perform all (or more than a desired threshold) number of soft linked practices contributing to that bundle. Considering the previously mentioned example of the thermal comfort bundle, this rule will allow certain practitioners to perform the bundle itself, while other practitioners will only be able to perform individual social practices such as using central heating and not the bundle itself.

Conceptualising practices influencing norms for performances: We had established before that practices evolve as a consequence of environmental influences, current trends and co-evolution of practices. Evolution of practices implies the evolution of elements they reference. E.g., between one time step and the next, the p_material of a particular practice agent might transform from 1001011001 to 1001000101 as a consequence of the three influences that acted upon the agent during that time step. This in turn impacts the calculation of similarity between elements in a future time step when a decision has to be made whether or not a practitioner is able to perform the concerned practice. This is our conceptualisation for practices influencing the norms for performances.

Conceptualising the co-evolution of practices (and bundles): We hypothesise that co-evolving practices will try to mirror all or parts of their elements - equivalent to sharing elements across practices. For instance, let us consider that at a particular time step the p_meaning element of the watching television practice is 1000110010 . If using central heating is a co-evolving practice, it will then try to mirror the $p \_$meaning element of the watching television practice as a consequence of co-evolution. This example is based on an empirical finding that people preferred to turn up the heating when watching TV with an intention of creating a comfortable and cosy ambience (i.e., meaning) $[8$, in review]. Co-evolution rules like these implemented in the model will allow practices to mirror the elements referenced by another, which in turn, will affect future performances of practices due to the similarity between elements rule.

Drawing together all the individual aspects described so far, we present our social practice theory based model conceptualisation in figure 3 . The flow of action between practitioner, practice and bundle agents are such that practitioners perform practices; practices come together to form bundles; both practices and bundles influence norms for the performance of practices. This is demonstrated in the middle portion of figure 3 . We have conceptualised this part with the intention of capturing the duality between practitioners and practices, i.e., practitioners perform practices, which in turn influence the norms for future performances.

The left portion of figure 3 shows the rules influencing practitioner agents and practice agents respectively. Our idea is that social influences and historical influences act upon practitioners (i.e. households) to enable them to draw elements together to perform practices. We recognise there may be other influences causing practitioners to perform practices, but here we have only considered these two. We consider three rules influencing practice agents: (i) update based on environmental influences, (ii) update based on current trends and (iii) update based on co-evolution. 
Lastly, the right portion of figure 3 depicts the input to the model and the output obtained from it. We conceptualise the input to the model as being any practitioner specific parameters. E.g., if households are considered as the practitioner agents, then household demographics (housing tenure and type, number of rooms, number of residents etc.) could be an input to the model. Output obtained from the model summarises the evolution of practices. This may be visualised as a growth trajectory of practices demonstrating the evolution of material, meaning and skill elements over time, as well as measuring the spread of practices among practitioners over time.

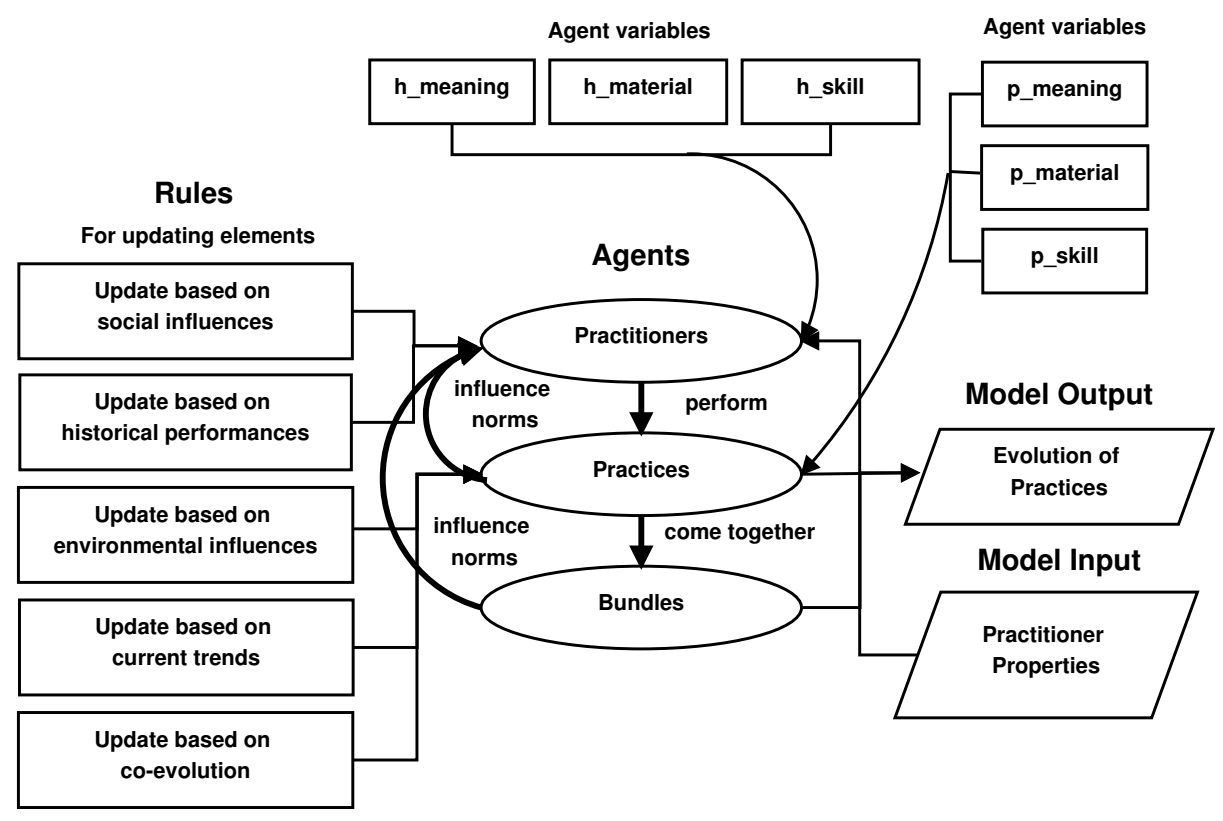

Fig. 3. Conceptualization of households performing social practices

\section{Conclusion and future work}

We have tried to achieve a three-fold outcome in this paper. Firstly, we have attempted to explicate in a systematic fashion the rich but dense concepts constituting the core principles of social practice theory. Second, to demonstrate the appropriateness of adopting a modelling position based on the theory of structuration to systematically conceptualise the key model processes. Lastly, to delineate an agent-based model conceptualisation to demonstrate the dynamics of processes associated with the production and re-production of social practices. It is our intention that the proposed model conceptualisation will allow 
formulations using suitable methods for pursuing specific case studies. Social practice theory is gaining popularity in many areas of research, so it is possible to adapt the model conceptualisation we have proposed here to investigate specific real-world phenomena. For instance, the authors are implementing the model to investigate the performances of energy intensive social practices in households.

Only few attempts have been made to conceptualise social practices using agent-based models. Holtz aimed at modelling the emergence of an abstract social practice based on the level of coherence achieved when material, meaning and competence elements come together [12]. He followed up with evidence to show that the proposed model reaches lock-in [13] quite early on in the simulation after which further changes to the social practice were blocked [14]. Balke et al. conceptualised social practices not as outcomes intended from the model but as agents themselves within the model. Their goal was to model households, social practices and industries as entities capable of shaping one another recursively, while also affecting the evolution of material artefacts within the model [15]. None of these, however, focused on all the three model processes that we have conceptualised here.

Besides, models aimed at simulating human societies often assume either an individualistic approach or a holistic approach. The former assumes that interactions between agents leads to the emergence of societal structure, while the latter considers that societal structure governs interaction between agents. But in the present case, the inherently recursive relationship between practitioners and practices motivated pursuing an alternative modelling strategy. We have followed Gilbert's recommendation of a modelling perspective that allows for duality to exist between structure and agency [16]. In one direction, practitioners act and their actions lead to the production and reproduction of practices and bundles. In the other direction, practices both enable and constrain the actions of practitioner agents. Gilbert notes that the ability to perceive and be responsive to macro-level structures is a notable capacity of humans and that it needs to be addressed in models synthesizing human societies [16]. Our conceptualisation of practitioners performing practices, which in turn influences the norms for future performances captures the duality between structure and agency in a realistic fashion.

\section{References}

1. Kuijer, S. C. (2014). Implications of social practice theory for sustainable design. Doctoral dissertation, TU Delft, Delft University of Technology. Netherlands.

2. Gilbert, N., \& Troitzsch, K. (2005). Simulation for the social scientist. McGraw-Hill International. New York.

3. Giddens, A. (1984). The constitution of society: Outline of the theory of structuration. Univ of California Press. Berkeley.

4. Shove, E., Pantzar, M., \& Watson, M. (2012). The dynamics of social practice: Everyday life and how it changes. London: SAGE Publications Ltd. 
5. Shove, E. (2005). Changing human behaviour and lifestyle: a challenge for sustainable consumption? Consumption Perspectives ecological economics. Cheltenham: Elgar pp. 111-132.

6. Reckwitz, A. (2002). Toward a theory of social practices a development in culturalist theorizing. European journal of social theory, 5(2), 243-263.

7. Wilhite, H. 2012. Towards a better accounting of the roles of body, things and habits in consumption in The Habits of Consumption: Studies across Disciplines in the Humanities and Social Sciences, ed. Warde, A., Southerton, D., Helsinki Collegium for Advanced Studies, Helsinki, pp. 87-99.

8. Roberts, T., Balke, T., \& Gilbert, N. Co-evolution of social practices: A case study of domestic energy use. (In Review) Energy Policy.

9. Gilbert, G. N. (2008). Agent-based models (No. 153). Sage. London.

10. Shove, E. (2003). Comfort, cleanliness and convenience: The social organization of normality. Oxford: Berg.

11. Macy, M. W., \& Willer, R. (2002). From factors to actors: Computational sociology and agent-based modeling. Annual review of sociology, 143-166.

12. Holtz, G. (2012). An agent-based model of social practices. Proceedings of the 8th Conference of the European Social Simulation Association, Salzburg.

13. Arthur, W. B. (1989). Competing technologies, increasing returns, and lock-in by historical events. The economic journal, 116-131.

14. Holtz, G. (2014). Generating social practices. Journal of Artificial Societies and Social Simulation, 17(1), 17.

15. Balke, T., Gilbert, N., Roberts, T., \& Xenitidou, M. (2014). Modelling energyconsuming social practices as agents. In Social Simulation Conference.

16. Gilbert, N., \& Conte, R. (1995). Artificial Societies: the computer simulation of social life. Taylor \& Francis, Inc. London. 